\title{
Gestational weight gain is an important risk factor for excessive fetal growth
}

Joung Min Lee, Min Jung Kim, Moon Young Kim, Jung Yeol Han, Hyun Kyeong Ahn, June Seek Choi, Jin Hoon Chung, Si Won Lee, You Jung Han, Dong Wook Kwak, Hyun Mee Ryu, Min Hyoung Kim

Department of Obstetrics and Gynecology, Cheil General Hospital and Women's Healthcare Center, Catholic Kwandong University College of Medicine, Seoul, Korea

\section{Objective}

To estimate the odds ratio of prepregnant body mass index (BMI), gestational weight gain (GWG), and gestational diabetes mellitus (GDM) for excessive fetal growth, which we define as large for gestational age (LGA).

\section{Methods}

We included 16,297 women who delivered a live-born singleton baby at term. We fit logistic regressions to estimate the odds ratios of variables, including maternal age, parity, prepregnant BMI $\geq 23$, GWG $\geq 15 \mathrm{~kg}$, and GDM, for LGA. We classified GWG into four categories $(<10,10-14.9,15-19.9$, and $\geq 20 \mathrm{~kg}$ ) and BMI into four categories (underweight, normal, overweight, and obese). After adjusting for age and parity, we analyzed the odds ratios of prepregnant BMI according to GWG between non-GDM and GDM women for LGA.

\section{Results}

The odds ratios of GWG $\geq 15 \mathrm{~kg}$ and prepregnancy BMI $\geq 23$ for LGA were 2.40 (95\% confidence interval [Cl], 2.162.67) and $2.24(95 \% \mathrm{Cl}, 1.99-2.51)$, respectively. The odd ratio of GDM was 1.37 (95\% Cl, 1.09-1.71). The risk of GDM women with normal/-overweight BMI and GWG $<15 \mathrm{~kg}$ for LGA was not significantly greater than those of the reference group. The odd ratios of GDM women with overweight/obese BMI and GWG 15 to $19.9 \mathrm{~kg}$ were $3.95(95 \%$ $\mathrm{Cl}, 1.26-12.38)$ and $9.70(95 \% \mathrm{Cl}, 3.79-24.87)$, respectively.

\section{Conclusion}

GWG $\geq 15 \mathrm{~kg}$ might be a more important risk factor for LGA than either prepregnancy BMI $\geq 23$ or GDM. Risk for LGA was highest in obese GDM women with GWG $\geq 15 \mathrm{~kg}$.

Keywords: Birth weight; Body mass index; Diabetes; Gestational; Weight gain

\section{Introduction}

Increasing obesity and excessive nutrition during pregnancy are important problems in both short- and long-term maternal and fetal health [1,2]. Being overweight or obese prior to pregnancy is associated with excessive fetal growth [3-6]. Excessive caloric intake during pregnancy results in increasing gestational weight gain (GWG). Previous studies reported that GWG is also associated with fetal growth [7-9]. The Institute of Medicine (IOM) recommended different GWGs according to the patient's prepregnant body mass index (BMI) for adequate fetal growth and reduction of pregnancy complications [10].

It is well established that gestational diabetes mellitus (GDM) increases the risk of adverse pregnancy outcomes, including being large for gestational age (LGA) and macrosomia [11,12]. Older maternal age and multiparity are also associated with a

Received: 2014.5.8. Revised: 2014.7.18. Accepted: 2014.8.11. Corresponding author: Min Hyoung Kim Department of Obstetrics and Gynecology, Cheil General Hospital and Women's Healthcare Center, Catholic Kwandong University College of Medicine, 17 Seoae-ro 1-gil, Jung-gu, Seoul 100-380, Korea Tel: +82-2-2000-7174 Fax: +82-2-2000-7183

E-mail: obdrmhk@naver.com

Articles published in Obstet Gynecol Sci are open-access, distributed under the terms of the Creative Commons Attribution Non-Commercial License (http://creativecommons. org/licenses/by-nc/3.0/) which permits unrestricted non-commercial use, distribution, and reproduction in any medium, provided the original work is properly cited.

Copyright (๑ 2014 Korean Society of Obstetrics and Gynecology 


\section{Obstetrics \& Gynecology Science}

Joung Min Lee, et al. Gestational weight gain and fetal growth

larger infant [13].

The influence of GDM, maternal BMI, and weight gain in pregnancy on excessive fetal growth varies by rate and ethnicity [14]. No prior studies have either analyzed the independent and combined risk factors for excessive fetal growth or compared the risk associated with equivalent prepregnant BMI and GWG for LGA in Korean women with and without GDM.

The objectives of this study were to evaluate the risk of age, parity, GDM, prepregnant BMI, and GWG for excessive fetal growth, defined as LGA. We also assessed detailed risk according to BMI and GWG for Korean women with and without GDM.

\section{Materials and methods}

We retrospectively reviewed the medical records of women who delivered a live-born singleton baby at term at a single medical center between 2010 and 2012. We excluded women with pre-existing medical conditions, such as pregestational diabetes and chronic hypertension. This study was approved by the ethics committee of Cheil General Hospital and Women's Health Care Center (CGH-IRB-2014-17).

Collected clinical characteristics of pregnant women included age, parity, prepregnant BMI $\left(\mathrm{kg} / \mathrm{m}^{2}\right), \mathrm{GWG}, \mathrm{GDM}$, gestational age at delivery, and delivery mode. Neonatal outcomes included birth weight, being LGA ( $\geq 90$ th percentile of gestational age) [15], and macrosomia ( $\geq 4.0 \mathrm{~kg}$ ).

Prepregnant BMI was calculated from maternal weight and height in the patient's first antenatal care interview; the women self-reported. We categorized them according to the World Health Organization definitions for weight: underweight, (BMI <18.5), normal $(18.5 \leq \mathrm{BMl}<23)$, overweight ( $23 \leq \mathrm{BMI}$ $<25)$, and obese (BMI $\geq 25$ ). We used the Carpenter-Coustan' criteria to diagnose GDM. GWG was defined as the difference between the patient's weight measured in the delivery unit and the prepregnant maternal weight recorded in the medical records at the first visit to the maternity health care center. We divided GWG into four categories: <10, 10 to $14.9,15$ to 19.9 , and $\geq 20 \mathrm{~kg}$.

Statistics included student's $t$-test for parametric and chisquare test for categorical variables for univariate analysis. We fit multiple logistic regressions to estimate the odds ratios on variables proven to be statistically significant for discriminating between LGA and non-LGA groups. To determine whether the association between GDM and infant size varied across categories of $\mathrm{BMI}$ and pregnancy weight gain, we divided the pregnant women into non-GDM and GDM groups and estimated the odds ratio for each category. Maternal age and parity were included as covariates in the adjusted analyses because they were regarded as potential confounding factors. The reference group was pregnant women without GDM, whose prepregnant BMI was normal and GWG was between 10 to $14.9 \mathrm{~kg}$. All statistical analyses were performed with SPSS ver. 16.0 (SPSS Inc., Chicago, IL, USA).

\section{Results}

We reviewed the medical records of 16,297 women who delivered a live-born singleton baby at term. Table 1 shows the maternal and neonatal characteristics of the study population. The prevalence of GDM in our study population was $4.5 \%$, the prevalence of overweight women (BMI $\geq 23$ ) was $10.1 \%$, and obesity prevalence $(\mathrm{BMI} \geq 25)$ was $8.5 \%$. The prevalence of GWG $\geq 15 \mathrm{~kg}$ was $36.1 \%$.

Table 1. Maternal and neonate characteristics of the study population $(n=16,297)$

\begin{tabular}{|lc|}
\hline \multicolumn{1}{c}{ Variable } & Value \\
\hline Age (yr) & $33.6 \pm 3.7$ \\
Nulliparity & $9,956(61.1)$ \\
Gestational diabetes mellitus & $727(4.5)$ \\
Prepregnancy body mass index ${ }^{\mathrm{a})}\left(\mathrm{kg} / \mathrm{m}^{2}\right)$ & \\
Underweight & $2,655(16.3)$ \\
Normal & $10,605(65.1)$ \\
Overweight & $1,645(10.1)$ \\
Obese & $1,392(8.5)$ \\
Gestational weight gain $(\mathrm{kg})$ & \\
$<10.0$ & $2,877(17.7)$ \\
10.0-14.9 & $7,538(46.3)$ \\
15.0-19.9 & $4,646(28.5)$ \\
$\geq 20.0$ & $1,236(7.6)$ \\
Gestational age at delivery (wk) & $39.4 \pm 1.1$ \\
Birth weight (g) & $3,290 \pm 393$ \\
Large for gestational age ${ }^{\text {b) }}$ & $1,634(10.0)$ \\
Macrosomiac) & $680(4.2)$ \\
\hline
\end{tabular}

Data are expressed as the mean \pm standard deviation or $\mathrm{n}(\%)$.

a) With World Health Organization-Asian criteria; ${ }^{b} \geq 90$ th percentile of gestational age; ${ }^{\text {c) Neonatal birth weight } \geq 4.0 \mathrm{~kg}}$ 


\title{
Obstetrics \& Gynecology Science
}

\author{
Vol. 57, No. 6, 2014
}

Comparison of clinical characteristics between LGA and non-LGA groups was expressed in Table 2. Maternal age in LGA group was older than that of non-LGA group. The prevalence of multiparity in LGA group was higher. Prepregnancy $\mathrm{BMI}$ and GWG in LGA group were higher than non-LGA group. GDM was more prevalent in LGA group. All characteristics were significantly different.

Table 3 shows the odds ratios from multiple logistic regression analyses for several variables and their association with LGA. We found that the following characteristics carried the increased risk for LGA: maternal age $\geq 35$ years, parity $\geq 1$, $G D M$, prepregnant $B M I \geq 23$, and $G W G \geq 15 \mathrm{~kg}$. The odd ratio of GWG $\geq 15 \mathrm{~kg}$ for $L G A$ was the highest .
The relationship between prepregnant BMI and GWG with excessive fetal growth between women with and without GDM is illustrated in Table 4. We used pregnant women with normal prepregnancy BMI and GWG between 10 to $14.9 \mathrm{~kg}$ who did not have GDM as the reference category. We adjusted for age and parity. The risk for LGA was greater with increased GWG in all BMI groups, regardless of GDM. In GDM women with normal and overweight BMI, the risk of LGA was not greater when GWG was $<15 \mathrm{~kg}$. When GWG was $15 \mathrm{~kg}$ or more, the risk in GDM women was greater than in nonGDM women, even when they had the same BMI. Risk for LGA was highest in obese GDM women with GWG $\geq 15 \mathrm{~kg}$.

Table 2. Clinical characteristics of $L G A^{\text {a) }}$ vs. non-LGA

\begin{tabular}{|lccc|}
\hline & Non-LGA $(\mathbf{n = 1 4 , 6 6 3 )}$ & LGA (n=1,634) & $\boldsymbol{P}$-value \\
\hline Age $(\mathrm{yr})$ & $33.6 \pm 3.7$ & $34.1 \pm 3.6$ & $<0.001$ \\
Multiparity & $5,555(37.9)$ & $786(48.1)$ & $<0.001$ \\
Prepregnancy BMI $\left(\mathrm{kg} / \mathrm{m}^{2}\right)$ & $20.8 \pm 2.7$ & $22.1 \pm 3.4$ & $<0.001$ \\
GWG $(\mathrm{kg})$ & $13.4 \pm 4.3$ & $15.3 \pm 4.9$ & $<0.001$ \\
Age $\geq 35 \mathrm{yr}$ & $5,105(34.8)$ & $655(40.1)$ & $<0.001$ \\
Prepregnancy BMI $\geq 23$ & $4,421(30.2)$ & $785(48.0)$ & $<0.001$ \\
GWG $\geq 15.0 \mathrm{~kg}$ & $5,026(34.3)$ & $856(52.4)$ & $<0.001$ \\
Gestational diabetes mellitus & $629(4.3)$ & $98(6.0)$ & 0.002 \\
Gestational age at delivery (wk) & $39.3 \pm 1.0$ & $39.6 \pm 1.0$ & $<0.001$ \\
Cesarean section & $5,047(34.4)$ & $834(51.0)$ & $<0.001$ \\
Birth weight $(\mathrm{g})$ & $3,212 \pm 325$ & $3,990 \pm 216$ & $<0.001$ \\
\hline
\end{tabular}

Data are expressed as the mean \pm standard deviation or $n(\%)$; Statistical analysis: Student's $t$-test and chi-square test.

LGA, large for gestational age; BMI, body mass index; GWG, gestational weight gain.

a) $\geq 90$ th percentile of gestational age

Table 3. Odd ratio of risk factors for large for gestational age ${ }^{a)}$

\begin{tabular}{|lccr|}
\hline Factors & Odds ratio & 95\% confidence interval & P-value \\
\hline Maternal age $\geq 35 \mathrm{yr}$ & 1.15 & $1.03-1.28$ & 0.012 \\
Parity $\geq 1$ & 1.48 & $1.33-1.65$ & $<0.001$ \\
Gestational diabetes mellitus & 1.37 & $1.09-1.72$ & 0.007 \\
Prepregnancy BMI ${ }^{\text {b) }} \geq 23$ & 2.24 & $1.99-2.52$ & $<0.001$ \\
GWG $\geq 15 \mathrm{~kg}$ & 2.40 & $2.16-2.67$ & $<0.001$ \\
\hline
\end{tabular}

Statistical analysis: binary and multiple logistic analyses.

BMI, body mass index; GWG, gestational weight gain.

a) $\geq 90$ th percentile of gestational age; ${ }^{\text {b) }}$ With WHO-Asian criteria. 


\section{Obstetrics \& Gynecology Science}

Joung Min Lee, et al. Gestational weight gain and fetal growth

Table 4. Associations of prepregnant body mass index and gestational weight gain with large for gestational age ${ }^{\text {a) }}$ between women without and with GDM

\begin{tabular}{|c|c|c|c|c|c|}
\hline & & & & & \\
\hline & & OR $(95 \% \mathrm{Cl})$ & $\mathrm{n}$ & OR $(95 \% \mathrm{Cl})$ & $\mathrm{n}$ \\
\hline Normal BMI ${ }^{b /}$ & & & & & \\
\hline GWG (kg) & $<10.0$ & $0.45(0.34-0.61)$ & 1,421 & $0.62(0.29-1.35)$ & 139 \\
\hline & $10.0-14.9$ & 1.00 (reference) & 4,811 & $1.18(0.68-2.03)$ & 161 \\
\hline & 15.0-19.9 & $1.69(1.45-1.97)$ & 3,153 & $4.00(2.20-7.27)$ & 60 \\
\hline & $\geq 20.0$ & $3.06(2.50-3.75)$ & 849 & $2.90(0.62-13.53)$ & 11 \\
\hline Overweight & & & & & \\
\hline GWG (kg) & $<10.0$ & $1.05(0.71-1.57)$ & 345 & $0.59(0.18-1.91)$ & 59 \\
\hline & $10.0-14.9$ & $1.62(1.25-2.11)$ & 636 & $0.71(0.17-2.98)$ & 34 \\
\hline & $15.0-19.9$ & $3.32(2.57-4.28)$ & 428 & $3.95(1.26-12.38)$ & 16 \\
\hline & $\geq 20.0$ & $5.67(3.80-8.46)$ & 122 & $3.05(0.34-27.48)$ & 5 \\
\hline Obese & & & & & \\
\hline GWG (kg) & $<10.0$ & $1.62(1.20-2.19)$ & 447 & $3.25(2.01-5.25)$ & 103 \\
\hline & $10.0-14.9$ & $2.69(2.08-3.49)$ & 452 & $4.05(2.17-7.55)$ & 53 \\
\hline & 15.0-19.9 & $3.54(2.53-4.95)$ & 216 & 9.70 (3.79-24.87) & 18 \\
\hline & $\geq 20.0$ & $5.96(3.82-9.30)$ & 95 & $6.66(1.21-36.74)$ & 6 \\
\hline
\end{tabular}

Statistical analysis: multiple logistic analyses.

GDM, gestational diabetes mellitus; OR, odds ratio; Cl, confidence interval; BMl, body mass index; GWG, gestational weight gain.

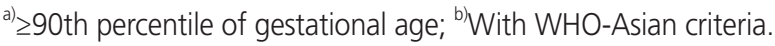

\section{Discussion}

In this study, we found that all the variables we investigated, including older maternal age, multiparity, prepregnant high BMI, GDM, and high GWG, increased the risk for LGA. Among these variables, high GWG had higher association with excessive fetal growth.

Many studies have reported an association of prepregnancy BMI and GWG with excessive fetal growth, either individually or combined, and most of these studies were analyses of women without GDM [16-19]. Dietz et al. [17] showed that high weight gain was associated with LGA and macrosomia and that the relationship was progressive. Ouzounian et al. [18] showed that prepregnancy weight and excessive weight gain are independent risk factors for macrosomia in women with GDM. In their study, GDM women with obesity and weight gain above the IOM threshold had increased risk for macrosomia that was six-fold above that for GDM women with normal weight who met IOM recommendations. Overweight GDM women who met IOM recommendations did not see an increased risk for macrosomia. In our study, we found an association between GWG and fetal growth, regardless of gestational diabetes, but the relationship was more prominent in GDM women.

In Asian women, Bowers et al. [13] found the strongest association was between GDM and LGA. In their study, Asian women with GDM, in combination with either high weight gain or obesity, had a five-fold increased risk for an LGA delivery. Black et al. [19] reported that, although being overweight and obese in the absence of GDM may substantially increase the risk of development of LGA, GDM in the absence of being overweight or obese does not appear to be associated with increased risk for LGA. They also observed that the prevalence of LGA was similar for overweight and normal-weight GDM women when GWG was $<18 \mathrm{~kg}$.

As we know from this study, GDM is a significant influencing factor of excessive fetal growth, albeit not an absolute one, and GWG, not GDM, is the most significant factor for fetal growth. Normal and overweight GDM women with GWG between 10 to $15 \mathrm{~kg}$ did not increase their risk for LGA compared to non-GDM normal weight women within the same range of GWG. However, obese women experienced 


\section{Obstetrics \& Gynecology Science}

Vol. 57, No. 6, 2014

an increased risk for LGA, although the GWG was less than $10 \mathrm{~kg}$ compared to normal-weight women with weight gain between 10 to $15 \mathrm{~kg}$. Obese GDM women had a higher risk for LGA at each category of GWG. Risk for LGA was highest in obese GDM women with GWG $\geq 15 \mathrm{~kg}$. Once GWG rose above $15 \mathrm{~kg}$, the risk for LGA increased significantly for all BMI categories, regardless of GDM status; however, the risk was greater in GDM cases with the same BMI.

Our results suggest a stronger relationship between maternal prepregnancy overweight/obesity and high weight gain with the occurrence of LGA in women with GDM than that in non-GDM women. These findings suggest that aggressive intervention before and during pregnancy could reduce the risk of high neonatal birth weight in GDM women. Recently, Park et al. [20] reported that minimal weight gain during pregnancy, below the IOM recommendation, may eliminate most adverse pregnancy outcomes, including LGA in obese GDM Asian women.

This study has several limitations. First, we had to use selfreported maternal weight because this was a retrospective study. Second, our study does not include other factors associated with birth weight, such as socioeconomic status, alcohol intake, and smoking during pregnancy; however, these risk factors are typically associated with lower birth weight [7]. Third, we did not classify GDM women according to whether they received insulin treatment.

Despite these limitations, the strengths of this study include the large sample size and that the results all come from a single institution with a comprehensive and consistent perinatal care program. Furthermore, our study is the first to report the combination risk from prepregnancy weight and degree of GWG for LGA between GDM and non-GDM women in Korea.

In conclusion GWG, along with prepregnancy BMI and GDM, is important risk factor for LGA. GWG $\geq 15 \mathrm{~kg}$ might be a more important risk factor for LGA than either prepregnancy $B M I \geq 23$ or GDM. Normal weight and overweight GDM women with GWG $<15 \mathrm{~kg}$ have no increased risk for excessive fetal growth compared to women in the same BMI, weight, and GWG categories who do not have GDM. Obese women with GDM and GWG $\geq 15 \mathrm{~kg}$ have the highest risk for excessive fetal growth. This study suggests that clinicians should show greater concern for appropriate weight gain during pregnancy.

\section{Conflict of interest}

No potential conflict of interest relevant to this article was reported.

\section{References}

1. Oken E, Kleinman KP, Belfort MB, Hammitt JK, Gillman $\mathrm{MW}$. Associations of gestational weight gain with shortand longer-term maternal and child health outcomes. Am J Epidemiol 2009;170:173-80.

2. Margerison Zilko CE, Rehkopf D, Abrams B. Association of maternal gestational weight gain with short- and long-term maternal and child health outcomes. Am J Obstet Gynecol 2010;202:574.e1-8.

3. Hayes M, Abrams B, Davidson EC. Influence of pregnancy weight on maternal and child health. Washington, DC: National Academies Press; 2007.

4. Oken E, Rifas-Shiman SL, Field AE, Frazier AL, Gillman MW. Maternal gestational weight gain and offspring weight in adolescence. Obstet Gynecol 2008;112:9991006.

5. Whitaker RC. Predicting preschooler obesity at birth: the role of maternal obesity in early pregnancy. Pediatrics 2004;114:e29-36.

6. Leddy MA, Power ML, Schulkin J. The impact of maternal obesity on maternal and fetal health. Rev Obstet Gynecol 2008;1:170-8.

7. Brawarsky P, Stotland NE, Jackson RA, Fuentes-Afflick E, Escobar GJ, Rubashkin N, et al. Pre-pregnancy and pregnancy-related factors and the risk of excessive or inadequate gestational weight gain. Int J Gynaecol Obstet 2005;91:125-31.

8. Rooney BL, Schauberger CW. Excess pregnancy weight gain and long-term obesity: one decade later. Obstet Gynecol 2002;100:245-52.

9. Young TK, Woodmansee B. Factors that are associated with cesarean delivery in a large private practice: the importance of prepregnancy body mass index and weight gain. Am J Obstet Gynecol 2002;187:312-8.

10. Rasmussen KM, Yaktine AL; Institute of Medicine. Weight gain during pregnancy: reexamining the guidelines. Washington, DC: The National Academy Press; 2009.

11. Ehrenberg HM, Mercer BM, Catalano PM. The influence 


\section{Obstetrics \& Gynecology Science}

Joung Min Lee, et al. Gestational weight gain and fetal growth

of obesity and diabetes on the prevalence of macrosomia. Am J Obstet Gynecol 2004;191:964-8.

12. Van Wootten W, Turner RE. Macrosomia in neonates of mothers with gestational diabetes is associated with body mass index and previous gestational diabetes. J Am Diet Assoc 2002;102:241-3.

13. Bowers K, Laughon SK, Kiely M, Brite J, Chen Z, Zhang C. Gestational diabetes, pre-pregnancy obesity and pregnancy weight gain in relation to excess fetal growth: variations by race/ethnicity. Diabetologia 2013;56:1263-71.

14. Silva JK, Kaholokula JK, Ratner R, Mau M. Ethnic differences in perinatal outcome of gestational diabetes mellitus. Diabetes Care 2006;29:2058-63.

15. Health Insurance Review and Assessment Service. Cesarean section assessment [Internet]. Seoul: Health Insurance Review and Assessment Service; 2010 [cited 2014 Oct 31]. Available from: http://hira.or.kr/cms/statistics.

16. Deierlein AL, Siega-Riz AM, Adair LS, Herring AH. Effects of pre-pregnancy body mass index and gestational weight gain on infant anthropometric outcomes. J Pediatr 2011;158:221-6.

17. Dietz PM, Callaghan WM, Sharma AJ. High pregnancy weight gain and risk of excessive fetal growth. Am J Obstet Gynecol 2009;201:51.e1-6.

18. Ouzounian JG, Hernandez GD, Korst LM, Montoro MM, Battista $L R$, Walden $C L$, et al. Pre-pregnancy weight and excess weight gain are risk factors for macrosomia in women with gestational diabetes. J Perinatol 2011;31:717-21.

19. Black MH, Sacks DA, Xiang AH, Lawrence JM. The relative contribution of prepregnancy overweight and obesity, gestational weight gain, and IADPSG-defined gestational diabetes mellitus to fetal overgrowth. Diabetes Care 2013;36:56-62.

20. Park JE, Park S, Daily JW, Kim SH. Low gestational weight gain improves infant and maternal pregnancy outcomes in overweight and obese Korean women with gestational diabetes mellitus. Gynecol Endocrinol 2011;27:775-81. 